\title{
REGISTROS DE ANFIBIOS EN EL BOSQUE NORPATAGÓNICO COSTERO DEL CANAL MESSIER, CHILE.
}

\author{
AMPHIBIAN RECORDS FROM THE COASTAL NOR - PATAGONIAN \\ FOREST ALONG THE MESSIER CHANNEL, CHILE.
}

\author{
Jessica Asencio ${ }^{1}$, Alejandro Kusch ${ }^{2,5}$, Juan M. Henríquez ${ }^{3,5}$ \& Jaime Cárcamo ${ }^{4}$
}

En Chile hay 57 especies de batracios, todos anuros, de los cuales sólo uno es introducido (Ortiz \& Díaz - Páez 2006). Éstos se distribuyen desde el nivel del mar hasta la cordillera, siendo algunos compartidos con los países limítrofes. De acuerdo a las especies existentes y su real distribución en la región de Magallanes existen discrepancias debido al poco conocimiento sobre el tema. Se han citado siete especies de batracios para la región (Venegas \& Sielfeld 1998) sin embargo, estudios recientes reconocen la existencia de sólo cuatro (Díaz-Páez \& Ortiz 2003). Por otra parte, los límites de distribución sur de las especies son poco exactos, como es el caso de Eupsophus calcaratus (Gunther, 1881) que la literatura especializada sitúa en el límite norte de la región (Formas et al. 1998). Gran parte de la batracofauna austral se distribuye en los canales patagónicos, generalmente en áreas de difícil acceso. Especies recientemente descritas han sido halladas mayoritariamente en la localidad de Puerto Edén (Formas et al. 1997, Formas et al. 1998). Sin embargo, la gran extensión del territorio austral, la escasez de colecciones y las diferencias en la información publicada hacen probable la existencia de especies no incluidas anteriormente en la batracrofauna de Magallanes y que se desconozca la distribución real de los anfibios en el extremo austral de Chile. El presente trabajo tiene como objetivo entregar nuevos registros de anfibios para Magallanes y además confirmar la presencia de Eusophus calcaratus.

El trabajo de campo se realizó en tres etapas (23 de noviembre a 5 de diciembre de 2006, entre el 16 y 20 de diciembre de 2007 y desde el 16 al 20 de enero de 2008), abarcando tres localidades de isla Wellington (Puerto Edén $49^{\circ} 09^{\prime} \mathrm{S}, 74^{\circ} 27^{\prime} \mathrm{W}$, bahía James $48^{\circ} 59^{\prime}$ S, $74^{\circ} 26^{\prime}$ W y bahía Broome $49^{\circ} 35^{\prime} \mathrm{S}, 74^{\circ} 43^{\prime} \mathrm{W}$ ) y dos localidades continentales (Libertad $48^{\circ} 88^{\prime} \mathrm{S}, 74^{\circ} 40^{\prime} \mathrm{W}$ y seno Edimburgo $49^{\circ} 05^{\prime}$ S, 74²2' W) (Fig. 1).

Durante la primera visita de terreno se utilizaron cuatro métodos de muestreo: búsqueda de refugio, parcelas, registro de encuentro visual (REV), y trampas de caída (Lips et al. 2001, DíazPáez et al. 2002). La búsqueda de refugio consistió en registrar anfibios en todos los hábitats hídricos,

\footnotetext{
Facultad de Ciencias, Universidad de Magallanes. jessi.asencio@gmail.com

Consultora Biota Austral Ltda., Casilla 19, Punta Arenas.

Laboratorio de Botánica, Instituto de la Patagonia, Universidad de Magallanes.

Laboratorio de Zoología, Instituto de la Patagonia, Universidad de Magallanes.

Centro de Ecología y Biodiversidad de la Patagonia Austral.
} 


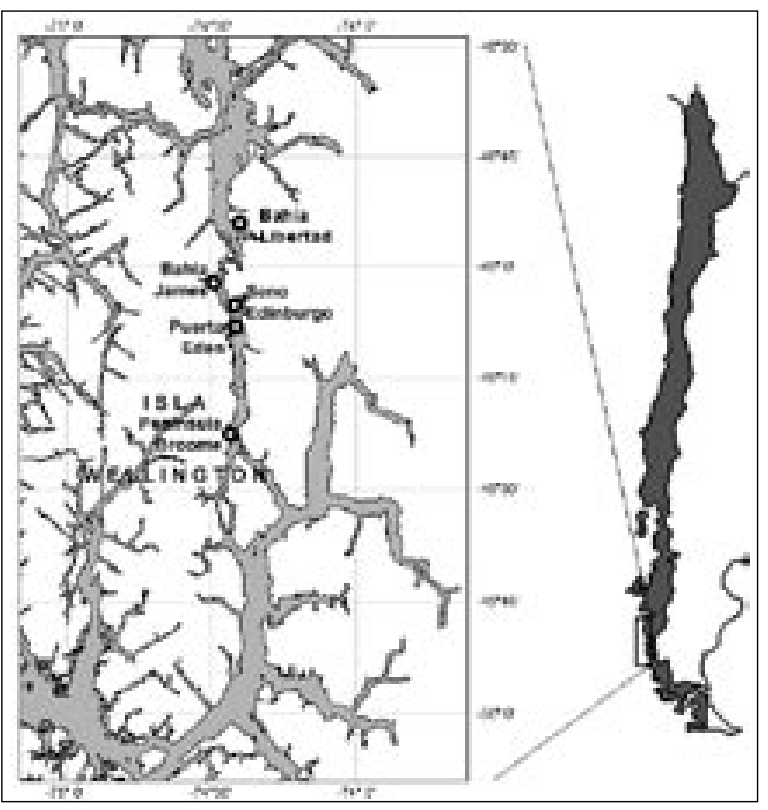

Fig. 1. Mapa del área de estudio donde se muestran en círculos los sitios de muestreo de anfibios.

bajo troncos caídos, entre la vegetación del suelo, lagunas, charcos y canales de drenaje en ambientes antrópicos. El área explorada se dividió en 10 parcelas de 3x3 metros dispuestas a lo largo de 5 transectos de 50 metros de longitud, establecidas en la turba y el bosque. El registro de encuentro visual consistió en caminar a lo largo de un transecto y buscar anfibios a una distancia visual de entre 1 a 3 metros a cada lado del camino dependiendo de la densidad vegetacional.
Las trampas de caída fueron agujeros en la tierra de $40 \times 40 \mathrm{~cm}$ de superficie y $40 \mathrm{~cm}$ de profundidad con agua, cada $5 \mathrm{~m}$ a lo largo de 10 transectos de 50 metros, los que fueron revisados cada 4 horas durante el día. Luego de tres días de muestreo se decidió utilizar sólo la búsqueda de refugio y el método REV debido a que las parcelas y trampas de caída resultaron ineficaces para lograr los objetivos. Esta experiencia negativa coincide con la obtenida por Díaz-Páez et al. (2002) en el PN Laguna San Rafael.

La identificación y edad relativa de los especímenes se basó en Cei (1962) y la colaboración de un especialista para la confirmación de las especies. Las medidas se hicieron con pie de metro $(0,05$ $\mathrm{mm}$ ) y balanza de precisión (tipo Pesola) de $30 \mathrm{gr}$ $\pm 0,5 \mathrm{gr}$.

Se encontraron 56 ejemplares que corresponden a 4 especies: Bufo variegatus (34 ejemplares), Eupsophus calcaratus (8), Batrachyla antartandica (11) y Alsodes australis (3). Datos morfométricos se presentan en la tabla 1.

Bufo variegatus (Günther, 1870)

Su distribución se extiende desde la región valdiviana hasta la provincia de Última Esperanza (Venegas \& Sielfeld 1998). Es común encontrarla en montículos de musgos o sobre piedras, pero siempre asociada a la comunidad de turberas (DíazPáez et al. 2002).

TABLA 1. Medidas promedio, desviación estándar y rango ( $\mathrm{mm}$ ) y peso (gr.) de los especímenes encontrados. Existen ejemplares depositados en el Pabellón de Colecciones del Instituto de la Patagonia, Universidad de Magallanes.

\begin{tabular}{|c|c|c|c|c|c|c|}
\hline & & & Talla $(\mathrm{mm})$ & & Peso (gr) & No.CZIP-B \\
\hline Especie & $\mathrm{N}$ & cabeza-urostilo & pata trasera & pata delantera & & \\
\hline $\begin{array}{l}\text { B. variegatus } \\
\text { (adulto) }\end{array}$ & 23 & $\begin{array}{c}31,3 \pm 5,87 \\
(16-41)\end{array}$ & $\begin{array}{c}32,6 \pm 6,99 \\
(15-41,5)\end{array}$ & $\begin{array}{c}18,6 \pm 4,03 \\
(7-24,5)\end{array}$ & $\begin{array}{c}3,0 \pm 1,2 \\
(0,8-7)\end{array}$ & $\begin{array}{c}15,16,19,21,22,23,26,28 \\
32,33,34,35,36,37,40,41 \\
\quad 42,43,45,46,47,48,49\end{array}$ \\
\hline $\begin{array}{l}\text { B. variegatus } \\
\text { (juvenil) }\end{array}$ & 4 & $\begin{array}{c}15,3 \pm 2,82 \\
(12-18)\end{array}$ & $\begin{array}{l}14,1 \pm 2,1 \\
(11-15,5)\end{array}$ & $\begin{array}{c}9,1 \pm 4,04 \\
(6-15)\end{array}$ & $\begin{array}{c}0,25 \pm 0,17 \\
(0,1-0,5)\end{array}$ & $17,20,25,27$ \\
\hline $\begin{array}{l}\text { B. antartandica } \\
\text { (adulto) }\end{array}$ & 4 & $\begin{array}{l}36,2 \pm 2,5 \\
(32-38,5)\end{array}$ & $\begin{array}{c}47,4 \pm 13,3 \\
(32-60)\end{array}$ & $\begin{array}{c}23,3 \pm 5,2 \\
(14-26,5)\end{array}$ & $\begin{array}{c}3,14 \pm 0,63 \\
(2,2-3,9)\end{array}$ & $24,29,30,31$ \\
\hline $\begin{array}{l}\text { B. antartandica } \\
\text { (juvenil) }\end{array}$ & 2 & $\begin{array}{c}20,3 \pm 1,5 \\
(19-22)\end{array}$ & $\begin{array}{c}27,1 \pm 2,7 \\
(24-29)\end{array}$ & $\begin{array}{c}13 \pm 1 \\
(12-14)\end{array}$ & $\begin{array}{c}0,83 \pm 0,21 \\
(0,6-1)\end{array}$ & 38,39 \\
\hline A. australis & 3 & $\begin{array}{c}51,1 \pm 9,26 \\
(41-58,5)\end{array}$ & $\begin{array}{c}72 \pm 11,35 \\
(59-80)\end{array}$ & $\begin{array}{c}31,83 \pm 7,28 \\
(25-39,5)\end{array}$ & 20,4 & $44,52,53$ \\
\hline E. calcaratus & 6 & $\begin{array}{c}36,38 \pm 4,09 \\
(29-41)\end{array}$ & $\begin{array}{c}52,18 \pm 8,94 \\
(34-63)\end{array}$ & $\begin{array}{c}19,58 \pm 4,81 \\
(13-21)\end{array}$ & $\begin{array}{l}4,8 \pm 0,9 \\
(4,2-5,5)\end{array}$ & $13,14,18,50,51,54$ \\
\hline
\end{tabular}


En nuestro estudio se registró la especie en 3 de las 4 localidades estudiadas (faltando en península Broome), con un total de 30 ejemplares adultos y 4 juveniles. Siempre se encontraron ejemplares solitarios y nunca relacionadas con otras especies. Esta especie fue la más frecuente en la zona de estudio, duplicando los encuentros en comparación con las otras.

Eupsophus calcaratus (Gunther, 1881)

La localidad típica es la Isla Grande de Chiloé con distribución en ambientes de influencia oceánica (Veloso \& Navarro 1988). Se le ha mencionado en Chile en las regiones de Los Ríos, Los Lagos y Aisén (Díaz-Páez \& Ortiz 2003). Formas et al. (1998) lo citan para Magallanes (seno Huemules) pero no se entregan localidades específicas por lo que podría tratarse de ejemplares colectados en la región de Aisén. No existen registros de colecciones de museo.

En el área de estudio se encontraron ocho ejemplares adultos (Fig. 2), dos ejemplares en seno Edimburgo, dos en bahía James y cuatro en bahía Broome, en bosque costero, bajo hojarasca, entre troncos húmedos y sobre musgos; similares ambientes a los del estudio realizado en el Parque Nacional Laguna San Rafael (Diaz-Paéz et al. 2002). Algunos de los ejemplares fueron encontrados junto a Alsodes australis.

\section{Batrachyla antartandica Barrio, 1967}

En Chile se conoce desde las regiones de Los Ríos, Los Lagos, Aisén y Magallanes (DíazPáez \& Ortiz 2003). En Magallanes esta especie fue registrada por Atalah \& Sielfeld (1976) en islas Virtudes (51 $31^{\prime} \mathrm{S}$; $74^{\circ} 55^{\prime} \mathrm{W}$ ) en ambientes de tundra magallánica.

Se registraron 8 ejemplares de esta especie, 1 en seno Edimburgo y 7 en Puerto Edén. La preferencia de microhábitat se asocia a charcos de agua, bajo troncos y pequeños riachuelos rodeados de vegetación densa de matorral y musgos.

\section{Alsodes australis (Formas et al., 1997)}

La especie fue descrita en base a ejemplares colectados en isla Wellington (Formas et al. 1997) y

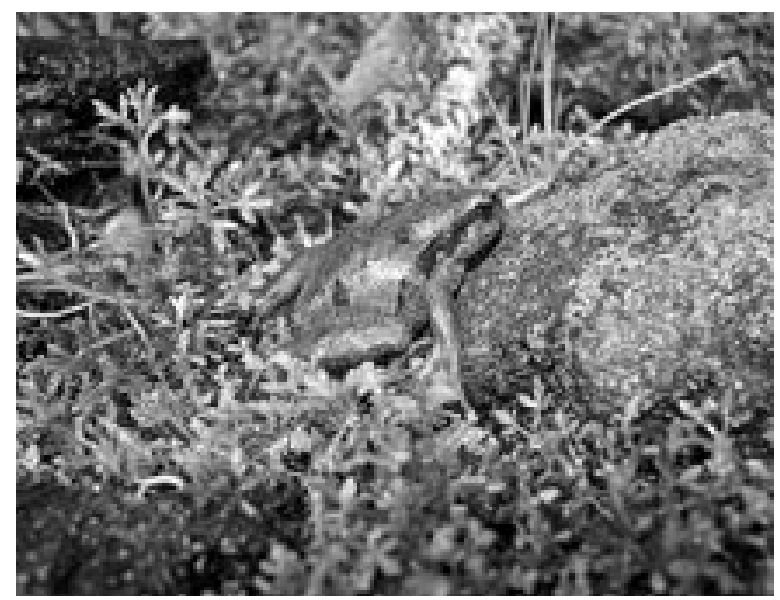

Fig. 2. Ejemplar de Eupsophus calcaratus colectado en bahía James (4859' S, 74²6’ W), isla Wellington.

no existen registros en otras localidades de la región de Magallanes. Díaz-Páez \& Ortiz (2003) sólo lo mencionan para la región de Aisén.

Se registró un ejemplar en Puerto Edén, específicamente en las inmediaciones de la Alcaldía de Mar de la Armada de Chile, entre restos de materiales de construcción y cercano a canales de drenaje. Otros dos ejemplares fueron colectados en península Broome bajo troncos en pastizal costero, también un sitio con cierto grado de intervención humana y sin mayor vegetación de gran altura.

En el trabajo de Cei (1962) se mencionan 4 especies para Magallanes, pero estudios recientes han descrito nuevas especies para la ciencia en los bosques Nor-patagónicos (e.g. Formas et al. 1997, Formas et al. 1998). Por otra parte, se desconocen los rangos de distribución de muchos anfibios en Magallanes, y el conocimiento sobre la biología y ecología es aún incipiente. Dos de los resultados más relevantes del presente estudio fueron: 1) la ausencia de registros de otras especies descritas exclusivamente para el área de estudio (e.g.: Alsodes kaweskarii, Atelognathus grandisonae) y 2) la confirmación de E. calcaratus para la región. Por otra parte, el registro de Atelognathus sp. en fiordo Toro, $53^{\circ} \mathrm{S}$, isla Riesco (Aguayo et al. 2007') refuerza la idea de profundizar estudios batracológicos en los canales patagónicos de Magallanes.

1 Aguayo, A. y colaboradores. 2007. Informe Final "Diagnóstico de la Flora y Fauna de vertebrados y Aves marinas del AMCP Francisco Coloane". Ministerio de Bienes Nacionales, Punta Arenas. 


\section{AGRADECIMIENTOS}

Este estudio fue posible gracias al financiamiento de la Dirección de Investigación de la Universidad de Magallanes al Programa de Investigación 217-01. Se agradece a las instituciones que apoyaron la logística de este proyecto, Naviera Navimag, Armada de Chile y Corporación Nacional Forestal (CONAF). Especialmente a la dotación de ALCAMAR Edén por su ayuda y hospitalidad. A la cooperación de la Dra. Helen Díaz por la información entregada y la revisión de fotografías y al Profesor Ramón Formas por enviar información. A Gloria Gallegos y Diego Machacan durante el trabajo en terreno. Agradecemos los comentarios de revisores anónimos que contribuyeron al mejoramiento en la versión preliminar de este texto. Al Servicio Agrícola y Ganadero (SAG) por el permiso de captura de investigación.

\section{LITERATURA CITADA}

Atalah. G. A. \& W. Sielfeld 1976. Presencia de Batrachyla antartandica Barrio en Magallanes. Anales Instituto Patagonia (Chile) 7: 169-170.

Cei, J. M. 1962. Batracios de Chile. Ediciones de la Universidad de Chile, Santiago.

Díaz - Páez, H., C. Williams \& R. A. Griffiths 2002. Diversidad y abundancia de anfibios en el Parque Nacional "Laguna San Rafael" (XI región, CHILE). Boletín del Museo Nacional de Historia Natural 51: 135-145.
Díaz - Páez, H. \& J. C. Ortiz 2003. Evaluación del estado de conservación de los anfibios en Chile. Revista Chilena de Historia Natural 76: $509-525$.

Formas, J. R., C. Úbeda, C. Cuevas \& J. Núñez 1997. Alsodes australis, a new species of Leptodactylidae frog from the temperate Nothofagus forest of southern Chile and Argentina. Studies in Neotropical Fauna \& Environment 32: 200-211.

Formas, J. R., C. Cuevas \& J. Núñez 1998. A new species of Alsodes (Amphibia: Anura: Leptodactylidae) from southern Chile. Proceedings of the Biological Society of Washington.

Lips, K. R, J. K Reaser, B. E Young \& R. Ibáñez 2001. Monitoreo de anfibios en América Latina: Manual de Protocolos. Society for the Study of Amphibians and Reptiles, Herpetological Circular 30: 1-115.

Ortiz, J. C. \& H. Díaz - Páez 2006. Estado de conocimiento de los anfibios de Chile. Gayana (Concepción) 70: 114 - 121.

Veloso, A. \& J. Navarro 1988. Lista Sistemática y distribución geográfica de anfibios y reptiles de Chile. Boll. Mus. Reg. Sci. Nat. Torino 6: $481-539$.

Venegas, C. \& W. Sielfeld 1998. Catálogo de los vertebrados de la región de Magallanes y Antártica Chilena. Ediciones de la Universidad de Magallanes, Punta Arenas. $122 \mathrm{pp}$. 